\title{
The Nuclear Astrophysics program at n_TOF (CERN)
}

N. Colonna ${ }^{1, *}$, O. Aberle ${ }^{8}$, J. Andrzejewski $^{7}$, L. Audouin ${ }^{16}$, M. Bacak ${ }^{11,8,12}$, J. Balibrea ${ }^{6}$, M. Barbagallo $^{1}$, F. Bečváŕ ${ }^{19}$, E. Berthoumieux ${ }^{12}$, J. Billowes ${ }^{17}$, D. Bosnar ${ }^{18}$, A. Brown ${ }^{20}, \mathrm{M}$. Caamaño $^{4}$, F. Calviño ${ }^{21}$, M. Calviani ${ }^{8}$, D. Cano-Ott ${ }^{6}$, R. Cardella $^{8}$, A. Casanovas $^{21}$, F. Cerutti $^{8}$, Y. H. Chen ${ }^{16}$, E. Chiaveri ${ }^{8}$, G. Clai ${ }^{15,30}$, G. Cortés ${ }^{21}$, M. A. Cortés-Giraldo ${ }^{2}$, Cosentino $^{22}$, S. Cristallo ${ }^{43}$, L. A. Damone ${ }^{1,23}$, M. Diakaki ${ }^{12}$, C. Domingo-Pardo ${ }^{3}$, L. R. Dressler $^{14}$, E. Dupont ${ }^{12}$, I. Durán ${ }^{4}$, B. Fernández-Domínguez ${ }^{4}$, A. Ferrari ${ }^{8}$, P. Ferreira ${ }^{10}$, P. Finocchiaro $^{22}$, V. Furman ${ }^{24}$, K. Göbel ${ }^{5}$, A. R. García ${ }^{6}$, A. Gawlik ${ }^{7}$, S. Gilardoni ${ }^{8}$, T. Glodariu $^{9}$, I. F. Gonçalves ${ }^{10}$, E. González ${ }^{6}$, E. Griesmayer ${ }^{11}$, C. Guerrero ${ }^{2}$, F. Gunsing ${ }^{12,8}$, H. Harada ${ }^{25}$, S. Heinitz ${ }^{14}$, A. Hernandez-Prieto ${ }^{21}$, J. Heyse ${ }^{26}$, D. G. Jenkins ${ }^{20}$, E. Jericha ${ }^{11}$, F. Käppeler ${ }^{27}$, Y. Kadi ${ }^{8}$, A. Kalamara ${ }^{28}$, P. Kavrigin ${ }^{11}$, A. Kimura ${ }^{25}$, N. Kivel ${ }^{14}$, M. Kokkoris $^{28}$, J. Kroll ${ }^{19}$, M. Krtička ${ }^{19}$, D. Kurtulgil ${ }^{5}$, C. Langer ${ }^{5}$, E. Leal-Cidoncha ${ }^{4}$, C. Lederer $^{29}$,J. Lerendegui-Marco ${ }^{2}$, H. Leeb ${ }^{11}$, S. Lo Meo ${ }^{15,30}$, S. J. Lonsdale ${ }^{29}$, S. Losito ${ }^{8}$, D.

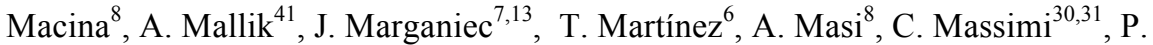
Mastinu $^{32}$, M. Mastromarco ${ }^{1}$, E. A. Maugeri ${ }^{14}$, A. Mazzone ${ }^{1,33}$, E. Mendoza ${ }^{6}$, A. Mengoni ${ }^{15}$, F. Mingrone ${ }^{8}$, P. M. Milazzo ${ }^{34}$, M. Mirea ${ }^{9}$, A. Musumarra ${ }^{22,35}$, A. Negret ${ }^{9}$, R. Nolte ${ }^{13}$, A. Oprea $^{9}$, N. Patronis ${ }^{36}$, A. Pavlik ${ }^{37}$, J. Perkowski ${ }^{7}$, L. Piersanti ${ }^{43}$, I. Porras ${ }^{38}$, J. Praena ${ }^{38}$, J. M. Quesada ${ }^{2}$, D. Radeck ${ }^{13}$, T. Rauscher ${ }^{39,40}$, R. Reifarth ${ }^{5}$, A. Riego ${ }^{21}$, M.S. Robles ${ }^{4}$, C. Rubbia $^{8}$, J. A. Ryan ${ }^{17}$ M. Sabaté-Gilarte ${ }^{8,2}$, A. Saxena ${ }^{41}$, P. Schillebeeckx ${ }^{26}$, D. Schumann ${ }^{14}$, P. Sedyshev ${ }^{24}$, A. G. Smith ${ }^{17}$, N. V. Sosnin ${ }^{17}$, A. Stamatopoulos ${ }^{28}$, G. Tagliente ${ }^{1}$, J. L. Tain $^{3}$, A. Tarifeño-Saldivia ${ }^{21}$ L. Tassan-Got ${ }^{16}$, A.Tsinganis ${ }^{8}$, S. Valenta ${ }^{19}$, G. Vannini ${ }^{30,31}$, V.

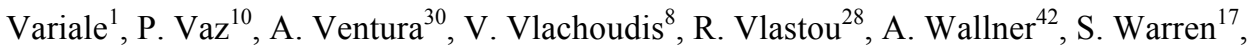
C. Weiss ${ }^{11}$, P. J. Woods ${ }^{29}$ T. Wright ${ }^{17}$, P. Žugec ${ }^{18,8}$

The $n$ TOF Collaboration

\footnotetext{
${ }^{I}$ Istituto Nazionale di Fisica Nucleare, Sezione di Bari, Italy; ${ }^{2}$ Universidad de Sevilla, Spain; ${ }^{3}$ Instituto de Fisica Corpuscular, Universidad de Valencia, Spain; ${ }^{4}$ University of Santiago de Compostela, Spain; ${ }^{5}$ Goethe University Frankfurt, Germany; ${ }^{6}$ Centro de Investigaciones Energéticas Medioambientales y Tecnológicas (CIEMAT), Spain; ${ }^{7}$ University of Lodz, Poland; ${ }^{8}$ European Organization for Nuclear Research (CERN), Switzerland; ${ }^{9}$ Horia Hulubei National Institute of Physics and Nuclear Engineering, Romania; ${ }^{10}$ Instituto Superior Técnico, Lisbon, Portugal;

${ }^{11}$ Technische Universität Wien, Austria $;{ }^{12}$ CEA Saclay, Irfu, Gif-sur-Yvette, France; ${ }^{13}$ PhysikalischTechnische Bundesanstalt (PTB), Braunschweig, Germany; ${ }^{14}$ Paul Scherrer Institut (PSI), Villingen, Switzerland; ${ }^{15}$ Agenzia nazionale per le nuove tecnologie (ENEA), Bologna, Italy; ${ }^{16}$ Institut de
}

${ }^{*}$ Corresponding author: nicola.colonna@ba.infn.it 
Physique Nucléaire, CNRS-IN2P3, Univ. Paris-Sud, Université Paris-Saclay, France, ${ }^{17}$ University of Manchester, United Kingdom; ${ }^{18}$ University of Zagreb, Croatia; ${ }^{19}$ Charles University, Prague, Czech Republic; ${ }^{20}$ University of York, United Kingdom; ${ }^{21}$ Universitat Politècnica de Catalunya, Spain; ${ }^{22}$ INFN Laboratori Nazionali del Sud, Catania, Italy; ${ }^{23}$ Dipartimento di Fisica, Università degli Studi di Bari, Italy; ${ }^{24}$ Joint Institute for Nuclear Research (JINR), Dubna, Russia; ${ }^{25}$ Japan Atomic Energy Agency (JAEA), Tokai-mura, Japan; ${ }^{26}$ European Commission, Joint Research Centre, Geel, Belgium; ${ }^{27}$ Karlsruhe Institute of Technology, IKP, Karlsruhe, Germany; ${ }^{28}$ National Technical University of Athens, Greece; ${ }^{29}$ School of Physics and Astronomy, University of Edinburgh, United Kingdom; ${ }^{30}$ Istituto Nazionale di Fisica Nucleare, Sezione di Bologna, Italy; ${ }^{31}$ Dipartimento di Fisica e Astronomia, Università di Bologna, Italy; ${ }^{32}$ Istituto Nazionale di Fisica Nucleare, Sezione di Legnaro, Italy; ${ }^{33}$ Consiglio Nazionale delle Ricerche, Bari, Italy; ${ }^{34}$ Istituto Nazionale di Fisica Nucleare, Sezione di Trieste, Italy; ${ }^{35}$ Dipartimento di Fisica e Astronomia, Università di Catania, Italy; ${ }^{36}$ University of Ioannina, Greece; ${ }^{37}$ University of Vienna, Faculty of Physics, Vienna, Austria; ${ }^{38}$ University of Granada, Spain; ${ }^{39}$ Department of Physics, University of Basel, Switzerland; ${ }^{40}$ Centre for Astrophysics Research, University of Hertfordshire, United Kingdom: ${ }^{41}$ Bhabha Atomic Research Centre (BARC), India $;{ }^{42}$ Australian National University, Canberra, ${ }^{43}$ INAF - Osservatorio Astronomico di Collurania, Teramo, Italy

\begin{abstract}
An important experimental program on Nuclear Astrophysics is being carried out at the $\mathrm{n}$-TOF since several years, in order to address the still open issues in stellar and primordial nucleosynthesis. Several neutron capture reactions relevant to s-process nucleosynthesis have been measured so far, some of which on important branching point radioisotopes. Furthermore, the construction of a second experimental area has recently opened the way to challenging measurements of (n, charged particle) reactions on isotopes of short half-life. The Nuclear Astrophysics program of the n_TOF Collaboration is here described, with emphasis on recent results relevant for stellar nucleosynthesis, stellar neutron sources and primordial nucleosynthesis.
\end{abstract}

\title{
1 Introduction
}

Neutron-induced cross sections play a fundamental role in Nuclear Astrophysics, being a key nuclear physics ingredient in stellar nucleosynthesis of heavy elements [1], as well as in light element production in Big Bang Nucleosynthesis [2]. In stars, neutron capture reactions are responsible for the production of the majority of elements heavier than $\mathrm{Fe}$, with two processes contributing more or less equally to the overall abundance pattern: the s- and the r-process. In the first one, which involves low neutron densities and mostly stable isotopes, progressively heavier elements are produced starting from the Fe seed nuclei, by neutron captures and subsequent $\beta$-decays. An interesting case occurs when an unstable nucleus with a relatively long half-life is produced in the process, as it can either decay or undergo a further neutron capture reaction, thus generating a branching in the s-process path. The competition between neutron capture and $\beta$-decay depends on the capture cross section, on the stellar half-life of the branching isotope and, most importantly, on the stellar thermodynamic conditions, in particular the neutron density and stellar temperature. As a consequence, the study of the branching points can shed some light on the stellar environment in which the s-process takes place, provided that accurate nuclear data on capture cross section and decay half-life are available.

Apart from heavy elements, neutron capture cross sections are needed for some light elements acting as neutron poison, or linked to stellar neutron sources. In this last case, neutron spectroscopy may provide information that are not easily accessible with other experimental techniques. Finally, neutron cross sections are needed to clarify one of the 
most intriguing and long-lasting problems in Big Bang Nucleosynthesis (BBN), i.e. the gross overestimate in BBN models of the primordial abundance of Lithium.

Although neutron-induced reactions of relevance for Nuclear Astrophysics are being studied since many decades at neutron facilities worldwide, some open issues in stellar and primordial nucleosynthesis still remain to be addressed in order to reach a more comprehensive understanding of the elemental abundance distribution and of the galactic chemical evolution. To this end, an intense experimental program is undergoing at the neutron facility n_TOF (CERN) since almost two decades, in order to reduce the uncertainty on neutron capture and ( $\mathrm{n}$, charged particle) cross sections for some key isotopes, and ultimately improve the reliability of astrophysical models. Thanks to the innovative characteristics of the neutron beam, to the development of high-performance detection and data acquisition systems, and to the use of refined analysis and simulation techniques, a number of valuable results of relevance for the field of Nuclear Astrophysics have been produced so far. After a brief description of the facility, the most important results recently obtained at $n \_$TOF are here discussed.

\section{The n_TOF facility at CERN}

The $\mathrm{n}_{-}$TOF facility was proposed and built at CERN with the aim of addressing the needs of new data of interest for Nuclear Astrophysics, for basic Nuclear Physics and for technological applications [3]. Neutrons are produced by spallation of a pulsed proton beam of $20 \mathrm{GeV} / \mathrm{c}$ momentum from the Proton Synchroton (PS) impinging on a monolithic water-cooled $\mathrm{Pb}$ target. A further layer of demineralized or borated water in the forward direction ensures additional moderation of the neutron energy, so to produce an almost isolethargic spectrum. For the first thirteen years of operation, only one neutron beam line, in the horizontal direction, was available, with the experimental area (EAR1) located at 185 $\mathrm{m}$ distance from the spallation target. In this area, the neutron beam is characterized by an instantaneous intensity of $10^{\circ}$ neutrons/bunch, an energy spectrum extending over almost eleven orders of magnitude, from thermal to $1 \mathrm{GeV}$, and a high energy resolution $(\Delta \mathrm{E} / \mathrm{E}<10$ ${ }^{3}$ in most of the energy range). More details on this area can be found in Ref. [4].

A second beam line in the vertical direction was recently completed at $n_{-}$TOF. The corresponding experimental area (EAR2) is located at $20 \mathrm{~m}$ distance from the spallation target [5]. Compared with EAR1, the neutron beam in the new area has a much higher intensity ( $>10^{\text {}}$ neutrons/bunch), at the expenses of a worse energy resolution and slightly smaller energy range. A schematic drawing of the facility is shown in Figure 1 (left panel), together with the neutron flux distribution in the two areas (right panel).

The two experimental areas are somewhat complementary. The characteristics of the neutron beam in EAR1 are ideal for high resolution measurements of neutron capture reactions on stable or long-lived radioactive isotopes $\left(t_{12}>100 \mathrm{y}\right)$, while the much higher flux in EAR2 allows one to perform measurements on radioisotopes with short half-life, on samples available in small (sub-mg) mass, on reactions with low cross section, or on all of these at the same time. In fact, for radioactive isotopes the combination of the higher flux and shorter time-of-flight results in an increase of the signal-to-background ratio of more than two orders of magnitude, relative to EAR1, when considering the background related to the radioactivity of the sample. As a consequence, in EAR2 it is possible to perform challenging measurements on isotopes with half-life as short as a few months. 

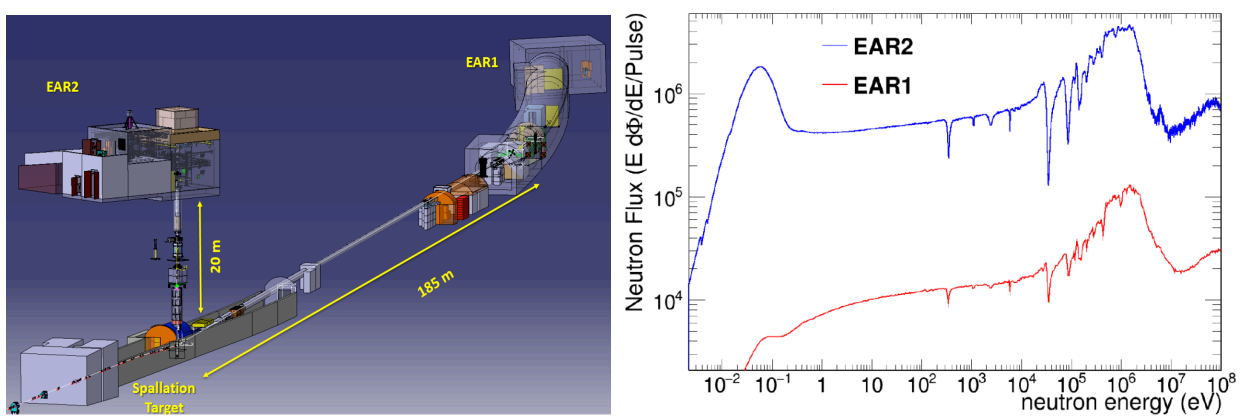

Fig. 1. Schematic drawing of the n_TOF facility, showing the horizontal and vertical beam lines and corresponding experimental areas (left panel) and, number of neutrons per bunch for the beams in the two areas (right panels).

\section{Recent results in Nuclear Astrophysics}

The experimental program of the n_TOF Collaboration in the field of Nuclear Astrophysics has, up to recently, mostly regarded neutron capture cross sections. Since the start of operation, in 2001, a large number of capture reactions have been measured, in EAR1, on a variety of subjects, from neutron magic nuclei, acting as bottleneck for the reaction flow of the s-process, to branching point isotopes, from light nuclei acting as neutron poison, to end-point nuclei, and, finally, to isotopes of special interest, such as those involved in the Os/Re nuclear cosmochronometer (see [6] for the full list of n_TOF results, or visit the site www.cern.ch/ntof). In all cases, a considerable reduction of the uncertainty on the capture cross section has been obtained, thanks to the peculiar features of the $\mathrm{n}_{-}$TOF neutron beam, in particular the high instantaneous neutron intensity and the high resolution. With the construction of the second experimental area, new lines of research have become accessible, in particular on reactions leading to light charged particle emission. In the following, a few indicative results recently obtained on some of the abovementioned classes of reactions are described more in details.

\subsection{Neutron capture cross section of branching point isotopes}

Branching points isotopes are unstable nuclei whose half-life is such that a competition exists between neutron capture and $\beta$-decay. An accurate knowledge of the capture cross section of these isotopes can help characterize the stellar conditions in which s-process occurs. A comprehensive list of the most important branching point isotopes can be found in [1].

At present, the neutron capture cross section of many of these isotopes is poorly known, mainly due to the difficulties in producing and handling samples of sufficient mass and adequate purity. Furthermore, the natural radioactivity of the sample is a source of background that might heavily affect the capture yield. For these reasons, few measurements have been performed on these isotopes so far, at a time-of-flight facility. At n_TOF, two important measurements, on "ism [7] and "Ni [8], were successfully completed in the first experimental area. The relatively long half-life and large sample masses resulted in a high signal-to-background ratio, and allowed the $\mathrm{n}$-TOF Collaboration to determine the Maxwellian Averaged Cross Section with an unprecedented few percent uncertainty. In both cases, the n_TOF results bore important Astrophysics implications.

The construction of the second experimental area at n_TOF, with the much larger neutron intensity, opened the way to measurements of neutron capture cross sections of 
isotopes with shorter half-life, and/or to samples of sub-mg masses. Three important sprocess branching point isotopes with these characteristics were recently measured at n_TOF: " $" \mathrm{Tm}$, $" \mathrm{Pm}$ and the ${ }^{20 \mathrm{Tl}}[$ [9]. Table I lists the half-life of these isotopes, together with the mass of the samples used in the $\mathrm{n}_{-} \mathrm{TOF}$ measurements, and the measured or theoretically calculated Maxwellian Averaged Cross Section.

Table 1. Measurements on the capture cross section of branching point isotopes measured so far at n_TOF. Experimental results are reported with the respective error, while for calculations a range of predicted values is indicated.

\begin{tabular}{|c|c|c|c|c|}
\hline Isotope & $\begin{array}{c}\text { Half-life } \\
\text { (years) }\end{array}$ & $\begin{array}{c}\text { Sample mass } \\
(\mathbf{m g})\end{array}$ & $\begin{array}{c}\text { Experimental } \\
\text { area }\end{array}$ & $\begin{array}{c}\text { MACS at 30 keV } \\
(\mathbf{m b})\end{array}$ \\
\hline${ }^{ } \mathrm{S} \mathrm{Sm}$ & 90. & 200. & EAR1 & $3100 \pm 160$ (Ref. [7]) \\
\hline${ }^{\circ} \mathrm{Ni}$ & 100. & 130. & EAR1 & $67 \pm 14$ (Ref. [8]) \\
\hline${ }^{20} \mathrm{Tl}$ & 3.8 & 11. & EAR1 & $97-328$ (calculated) \\
\hline${ }^{12} \mathrm{Tm}$ & 1.9 & 3.6 & EAR1 \& EAR2 & $243-1332$ (calculated) \\
\hline${ }^{\circ} \mathrm{Pm}$ & 2.6 & 0.3 & EAR2 & $\begin{array}{c}400-2000 \text { (calculated) } \\
709 \pm 100 \text { (Ref. [9]) }\end{array}$ \\
\hline
\end{tabular}

The " Tm branching point is independent of stellar temperature and can therefore provide a strong constraint on the s-process neutron density in low mass AGB stars, while ${ }^{20} \mathrm{Tl}$, responsible for the production of ${ }^{202 a s} \mathrm{~Pb}$ (screened from the r-process by the stable ${ }^{2 n} \mathrm{Hg}$ and ${ }^{20} \mathrm{Tl}$ isotopes), is crucial for understanding the nucleosynthesis of heavy elements in AGB stars, as well as for providing hints on the stellar object that polluted the proto-solar nebula at the epoch of the solar system formation. Finally, the analysis of the branching point isotope $\mathrm{Pm}$ in the $\mathrm{Nd}-\mathrm{Pm}-\mathrm{Sm}$ region is important for modeling the AGB star evolution and to put accurate constraints on the interplay between metallicity, initial stellar mass and mixing processes.

Measuring the neutron capture cross section of these three isotopes is a challenging task because of the high specific activity of these radionuclides, typically resulting in a poor signal-to-background ratio, and because of the difficulty in the procurement of a sufficient amount of material with adequate purity, as well as in sample preparation and characterization. Because of these problems, very scarce or no data have been collected so far on these isotopes, and at present one can only rely on highly uncertain theoretical calculations, as reported in Table I, or on a single activation measurement for "Pm [10]. The availability of intense neutron beams at $n \_$TOF finally provided the unique opportunity to collect, for the first time, high quality data at a time-of-flight installation for these reactions. The radioisotopes were produced at the Institute Laue Langevin ILL (Grenoble, France) high flux reactor, by irradiating for several weeks under the thermal neutron beam the stable progenitors with thermal neutrons. The chemical separation and sample preparation was carried out at the Paul Scherrer Institute (PSI), Switzerland, as described in Ref. [11].

The analysis of these reactions is currently under way, but once completed it is expected to provide important experimental information on the capture cross section of these isotopes, in some cases for the first time ever, possibly leading to a better characterization of these branching points.

\subsection{Studies of stellar neutron sources by neutron spectroscopy}

One of the main issues in Nuclear Astrophysics regards the modelling of neutron generation in the stellar environment. Large efforts are currently being devoted in various 
laboratories worldwide to determine the reaction rate of the two most important neutron sources in AGB stars, i.e. the " $\mathrm{C}(\alpha, \mathrm{n}){ }^{1} \mathrm{O}$ and ${ }^{2} \mathrm{Ne}(\alpha, \mathrm{n}){ }^{3} \mathrm{Mg}$ reaction. The accurate knowledge of the reaction rate for these sources is decisive for deriving information on neutron density, temperature, and pressure in the He-burning layers of AGB stars. At low projectile energy, direct measurements are difficult and yield, at present, highly uncertain results. An alternative approach to constrain the reaction rate at low energy is to measure indirect and transfer reactions. In particular, the study of neutron-induced reactions could provide useful information on the properties of the states above the neutron threshold of the compound nuclei formed in the reactions.

In this respect, neutron spectroscopy of ${ }^{20} \mathrm{Mg}$ states, of relevance for the ${ }^{2} \mathrm{Ne}(\alpha, \mathrm{n}){ }^{2} \mathrm{Mg}$ neutron source, was recently carried out using data from neutron capture and transmission measurements performed at $n_{-}$TOF and GELINA, respectively [12]. This latter facility, located at the European Commission Joint Research Center in Geel, Belgium, is based on a linear electron accelerator and a mercury-cooled uranium target for neutron production [13]. The time properties of the electron beam and the small target size make GELINA particularly suited for high-resolution cross section measurements in the resonance region. A ${ }^{6}$ Li-glass detector was used in the transmission measurement, performed in the experimental station placed at $50 \mathrm{~m}$ distance from the neutron-producing target.

High-resolution neutron capture yields for a high-purity, metallic ${ }^{25} \mathrm{Mg}$ sample measured in EAR1 at $n \_$TOF, were combined with the transmission data collected at GELINA in a simultaneous R-matrix analysis performed on both data sets. This allowed to univocally assign the spin and parity of some important excited states of ${ }^{26} \mathrm{Mg}$ with natural parity just above the neutron separation energy, not easily accessible in the direct $\alpha+\mathrm{Ne}$ reactions. Based on the $n_{-}$TOF/GELINA data, a new, more reliable estimate of the ${ }^{2} \mathrm{Ne}(\alpha, \mathrm{n})$ cross section below $800 \mathrm{keV} \alpha$-particle energy was obtained. The result, shown Figure 2, puts firmer constraints on the reaction rate of this important stellar neutron source. The use of neutron spectroscopy is now being considered at n_TOF for the primary neutron source as well, the " $\mathrm{C}(\alpha, \mathrm{n}) \mathrm{O}$ one. In this case, the Collaboration is planning a measurement with high resolution of the time-reversal reaction, the " $\mathrm{O}(\mathrm{n}, \alpha) \mathrm{C}$.

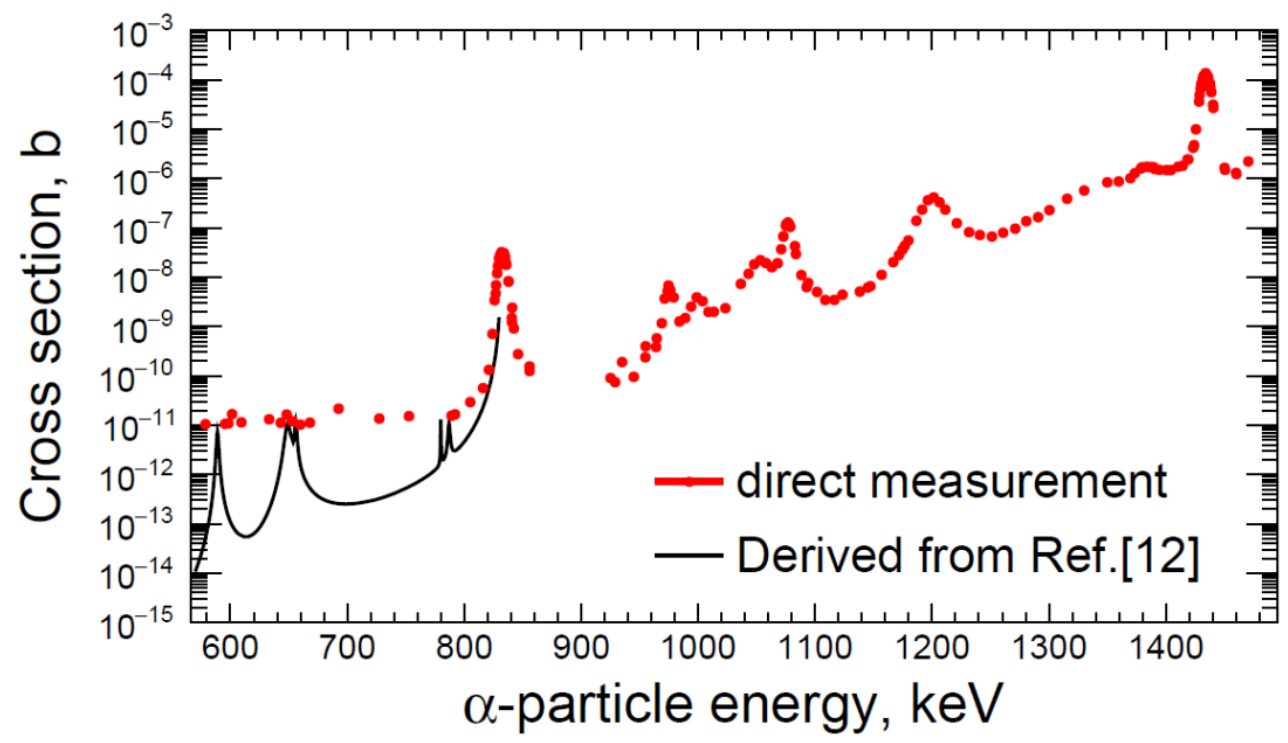


Fig. 2. Cross section of the ${ }^{2} \mathrm{Ne}(\alpha, \mathrm{n})^{2} \mathrm{Mg}$ reaction from a direct measurement (red symbols) and calculated on the basis of the neutron spectroscopy of Ref. [12].

\subsection{Measurement of $(n, c p)$ reactions in EAR2@n_TOF}

The very high instantaneous neutron intensity in the second experimental area at $\mathrm{n} \_$TOF has also made feasible to measure ( $\mathrm{n}$, charged particle) reactions of low cross section and/or for isotopes of short-half life and/or with samples of extremely small mass. Two isotopes of high relevance in astrophysics reactions have been investigated so far at n_TOF: $\mathrm{Be}$ and ${ }^{\circ \mathrm{Al}}$.

Neutron-induced reactions on $\mathrm{Be}\left(\mathrm{t}_{12}=53.2\right.$ days $)$ are of interest for Big Bang Nucleosynthesis, more specifically for the so-called "Cosmological Lithium problem" [2]. More details on the motivation and on the measurements can be find in [14-15]. Two reactions were measured on this isotope at n_TOF: the $\operatorname{Be}(n, \alpha) \cdot \mathrm{He}$ and the $\mathrm{Be}(\mathrm{n}, \mathrm{p}) \mathrm{Li}$ reactions. For the first one, never measured before above the thermal neutron energy, two samples of $\sim 3 \mu \mathrm{g}$ total mass were used (corresponding to a total activity of $40 \mathrm{GBq}$ ). A sufficient amount of 'Be was extracted from the SINQ spallation source of PSI, chemically separated and deposited on different backings using different techniques. More details on the sample preparation can be found in [16]. For the $(n, p)$ reaction, characterized by a much higher cross section, it was sufficient to use a sample of mass as small as $90 \mathrm{ng}$. In this last case, however, a sample of high isotopic purity was produced by implantation of a $\mathrm{Be}$ beam at ISOLDE. High performance experimental setups, based on solid state detectors [17-18], were specifically designed and built for the measurements. Combined with the characteristics of the EAR2 neutron beam, the world-class expertise in sample preparation and detector development has led the n_TOF Collaboration to collect high quality results on both reactions, which are expected to clarify once and for all the role of the neutron induced reactions on $\mathrm{Be}$ in the Cosmological Lithium Problem.

Other successful measurements recently performed at n_TOF regard the ${ }^{\circ} \mathrm{Al}(\mathrm{n}, \mathrm{p})$ and $(n, \alpha)$ reactions [19]. Precise satellite observations of this cosmic $\gamma$-ray emitter, whose relatively short lifetime (1 My) provides evidence of ongoing nucleosynthesis in our galaxy, offer the opportunity to improve calculations of the production and destruction rates of some key astrophysical reaction. However, the poor knowledge of the (n, cp) reactions on ${ }^{2} \mathrm{Al}$, responsible for its destruction, are at present the major source of uncertainty in predicting the amount of ${ }^{20} \mathrm{Al}$ ejected into the interstellar medium by Wolf-Rayet stars in the stellar wind or following the supernova explosion. The challenge in this case is related to the availability of a sufficient amount of ${ }^{20} \mathrm{Al}$, and to the need of identifying the emitted particles. At n_TOF, a sample of $10 \mu \mathrm{g}$ mass, produced at Los Alamos National Laboratory (USA) [20] was irradiated under the high-flux neutron beam of the second experimental area, with the reaction product detected in a position-sensitive solid state telescope. While the analysis is still in progress, preliminary results indicate that the n_TOF measurement might provide accurate new data on the important $n+{ }^{26} \mathrm{Al}$ reaction cross sections.

\section{Conclusions and perspectives}

The $\mathrm{n} \_$TOF Collaboration has been carrying out since several years an ambitious experimental program on Nuclear Astrophysics, with the aim of reducing the uncertainties on cross sections of various reactions involved in stellar and primordial nucleosynthesis. The unique features of the neutron beam in the two experimental areas have made possible to study neutron capture reactions on several unstable isotopes acting as branching points, 
to perform neutron spectroscopy relevant for stellar neutron sources and, more recently, to measure challenging ( $\mathrm{n}, \mathrm{cp}$ ) reactions on short-lived radioisotopes or sub-mg samples. In all cases, a substantial reduction of the uncertainties on cross sections relevant for stellar and primordial nucleosynthesis has been achieved. New, challenging measurements are foreseen for the near future, whose results are expected to lead to significant advancement in the field of Nuclear Astrophysics.

We acknowledge the support by the Narodowe Centrum Nauki (NCN), under the Grant No. UMO2012/04/M/ST2/00700.

\section{References}

1. F. Käppeler et al., Rev. Mod. Phys 83, 157 (2011)

2. R.H. Cyburt et al., Phys. Rev. D 69, 123519 (2004)

3. C. Rubbia et al, Tech Rep. CERN/LHAC/98-02 CERN (1998)

4. C. Guerrero et al., Eur. Phys J. A 49, 27 (2013)

5. C. Weiss et al., Nucl. Instrum. Methods A 799, 90 (2015)

6. F. Gunsing et al., Eur. Phys. J. Plus 131, 371 (2016)

7. U. Abbondanno et al., Phys. Rev. Lett. 93, 161103 (2004)

8. C. Lederer et al., Phys. Rev. Lett. 110, 022501 (2013)

9. C. Guerrero et al., JPS Conf. Proc. 14, 010903 (2016)

10. C. Arlandini, M. Heil, R. Reifarth, F. Käppeler, P.V. Sedyshev, Nucl. Phys. A 688, 487c (2001)

11. S. Heinitz et al., Radiochemica Acta, DOI: 10.1515/ract-2016-2728

12. C. Massimi et al., Phys. Lett. B 768, 1 (2017)

13. W. Mondelaers and P. Schillebeeckx, Notiziario Neutroni e Luce di Sincrotrone 11, 19 (2006)

14. M. Barbagallo et al., Phys. Rev. Let. 117, 152701 (2016);

15. M. Barbagallo et al., contribution to NPA VIII

16. E.A. Maugeri et al., J. Inst. 12, P02016 (2017)

17. L. Cosentino et al., Nucl. Instrum. Methods A 830, 197 (2016)

18. M. Barbagallo et al., submitted to Nucl. Instrum. Methods A

19. C. Lederer et al., CERN-INTC-20154-006/INTC-P-406

20. C. Ingelbrecht et al., Nucl. Instr. Meth. A 480, 114 (2002) 